\title{
Integral formula of Minkowski type and new characterization of the Wulff shape
}

\author{
Yijun $\mathrm{He}^{*} \quad$ Haizhong $\mathrm{Li}^{\dagger}$
}

\begin{abstract}
Given a positive function $F$ on $S^{n}$ which satisfies a convexity condition, we introduce the $r$-th anisotropic mean curvature $M_{r}$ for hypersurfaces in $\mathbb{R}^{n+1}$ which is a generalization of the usual $r$-th mean curvature $H_{r}$. We get integral formulas of Minkowski type for compact hypersurfaces in $R^{n+1}$. We give some new characterizations of the Wulff shape by use of our integral formulas of Minkowski type, in case $F=1$ which reduces to some well-known results.
\end{abstract}

2000 Mathematics Subject Classification: Primary 53C42, 53A30; Secondary $53 \mathrm{~B} 25$.

Key words and phrases: Wulff shape, F-Weingarten operator, anisotropic principal curvature, $r$-th anisotropic mean curvature, integral formula of Minkowski type.

\section{$\S 1 \quad$ Introduction}

Let $F: S^{n} \rightarrow \mathbb{R}^{+}$be a smooth function which satisfies the following convexity condition:

$$
\left(D^{2} F+F 1\right)_{x}>0, \quad \forall x \in S^{n},
$$

where $D^{2} F$ denotes the intrinsic Hessian of $F$ on $S^{n}$ and 1 denotes the identity on $T_{x} S^{n},>0$ means that the matrix is positive definite. We consider the map

$$
\begin{gathered}
\phi: S^{n} \rightarrow \mathbb{R}^{n+1}, \\
x \rightarrow F(x) x+\left(\operatorname{grad}_{S^{n}} F\right)_{x},
\end{gathered}
$$

\footnotetext{
*Partially supported by Tianyuan Fund for Mathematics of NSFC.

${ }^{\dagger}$ Partially supported by the grant No. 10531090 of the NSFC and by Doctoral Program Foundation of the Ministry of Education of China (2006).
} 
its image $W_{F}=\phi\left(S^{n}\right)$ is a smooth, convex hypersurface in $\mathbb{R}^{n+1}$ called the Wulff shape of $F$ (see [3], [6], [10], [13], [14]).

Now let $X: M \rightarrow \mathbb{R}^{n+1}$ ba a smooth immersion of a compact, orientable hypersurface without boundary. Let $\nu: M \rightarrow S^{n}$ denotes its Gauss map, that is, $\nu$ is an unit inner normal vector of $M$.

Let $A_{F}=D^{2} F+F 1, S_{F}=-A_{F} \circ \mathrm{d} \nu . S_{F}$ is called the $F$-Weingarten operator, and the eigenvalues of $S_{F}$ are called anisotropic principal curvatures. Let $\sigma_{r}$ be the elementary symmetric functions of the anisotropic principal curvatures $\lambda_{1}, \lambda_{2}, \cdots, \lambda_{n}$ :

$$
\sigma_{r}=\sum_{i_{1}<\cdots<i_{r}} \lambda_{i_{1}} \cdots \lambda_{i_{r}} \quad(1 \leq r \leq n)
$$

We set $\sigma_{0}=1$. The $r$-anisotropic mean curvature $M_{r}$ is defined by $M_{r}=\sigma_{r} / C_{n}^{r}$.

In this paper we first give the following integral formulas of Minkowski type for compact hypersurfaces in $\mathbb{R}^{n+1}$.

Theorem 1.1. Let $X: M \rightarrow \mathbb{R}^{n+1}$ be an $n$-dimensional compact hypersurface, $F: S^{n} \rightarrow$ $\mathbb{R}^{+}$be a smooth function which satisfies (1), then we have the following integral formulas of Minkowski type hold:

$$
\int_{M}\left(F M_{r}+M_{r+1}\langle X, \nu\rangle\right) \mathrm{d} A_{X}=0, \quad r=0,1, \cdots, n-1 .
$$

By use of above integral formulas of Minkowski type, we prove the following new characterizations of the Wulff shape:

Theorem 1.2. Let $X: M \rightarrow \mathbb{R}^{n+1}$ be an $n$-dimensional compact hypersurface, $F: S^{n} \rightarrow$ $\mathbb{R}^{+}$be a smooth function which satisfies (1), and $M_{1}=$ const and $\langle X, \nu\rangle$ has fixed sign, then up to translations and homotheties, $X(M)$ is the Wulff shape.

Theorem 1.3. Let $X: M \rightarrow \mathbb{R}^{n+1}$ be an $n$-dimensional compact hypersurface, $F: S^{n} \rightarrow$ $\mathbb{R}^{+}$be a smooth function which satisfies (1). If $M_{1}=$ const and $M_{r}=$ const for some $r, 2 \leq r \leq n$, then up to translations and homotheties, $X(M)$ is the Wulff shape.

Theorem 1.4. Let $X: M \rightarrow \mathbb{R}^{n+1}$ be an $n$-dimensional compact convex hypersurface, $F: S^{n} \rightarrow \mathbb{R}^{+}$be a smooth function which satisfies (1). If $\frac{M_{r}}{M_{k}}=$ const for some $k$ and $r$, with $0 \leq k<r \leq n$, then then up to translations and homotheties, $X(M)$ is the Wulff shape.

Theorem 1.5. Let $X: M \rightarrow \mathbb{R}^{n+1}$ be an $n$-dimensional compact hypersurface, $F: S^{n} \rightarrow$ $\mathbb{R}^{+}$be a smooth function which satisfies (1). If $\frac{M_{k}}{M_{n}}=$ const for some $k$, with $0 \leq k \leq$ $n-1$, then then up to translations and homotheties, $X(M)$ is the Wulff shape. 
Choosing $k=0$ in Theorem 1.4, we get

Corollary 1.1 Let $X: M \rightarrow \mathbb{R}^{n+1}$ be an $n$-dimensional compact convex hypersurface, $F: S^{n} \rightarrow \mathbb{R}^{+}$be a smooth function which satisfies (1), and for a fixed $r$ with $1 \leq r \leq n$, $M_{r}=$ const, then up to translations and homotheties, $X(M)$ is the Wulff shape.

Remark 1.1 When $F=1$, Wulff shape is just the round sphere and $M_{r}=H_{r}$, formula (2) reduces to the classical Minkowski integral formula (see [5] or [11]), Theorem 1.2 reduces to the classical Theorem given by Süss [12], Corollary 1.1 reduces to Theorem of Yano [15], Theorem 1.3 reduces to Theorem of Choe [2].

\section{$\S 2 \quad$ Preliminaries}

Let $\left\{E_{1}, \cdots, E_{n}\right\}$ is a local orthogonal frame on $S^{n}$, let $e_{i}=E_{i} \circ \nu$, where $i=$ $1, \cdots, n$, then $\left\{e_{1}, \cdots, e_{n}\right\}$ is a local orthogonal frame of $X: M \rightarrow \mathbb{R}^{n+1}$.

The structure equation of $S^{n}$ is:

$$
\left\{\begin{array}{l}
\mathrm{d} x=\sum_{i} \theta_{i} E_{i} \\
\mathrm{~d} E_{i}=\sum_{j} \theta_{i j} E_{j}-\theta_{i} x \\
\mathrm{~d} \theta_{i}=\sum_{j} \theta_{i j} \wedge \theta_{j} \\
\mathrm{~d} \theta_{i j}-\sum_{k} \theta_{i k} \wedge \theta_{k j}=-\frac{1}{2} \sum_{k l} \tilde{R}_{i j k l} \theta_{k} \wedge \theta_{l}=-\theta_{i} \wedge \theta_{j}
\end{array}\right.
$$

where $\theta_{i j}+\theta_{j i}=0$ and

$$
\tilde{R}_{i j k l}=\delta_{i k} \delta_{j l}-\delta_{i l} \delta_{j k}
$$

The structure equation of $X$ is (see [7], [8]):

$$
\left\{\begin{array}{l}
\mathrm{d} X=\sum_{i} \omega_{i} e_{i} \\
\mathrm{~d} \nu=-\sum_{i j} h_{i j} \omega_{j} e_{i} \\
\mathrm{~d} e_{i}=\sum_{j} \omega_{i j} e_{j}+\sum_{j} h_{i j} \omega_{j} \nu \\
\mathrm{d} \omega_{i}=\sum_{j} \omega_{i j} \wedge \omega_{j} \\
\mathrm{~d} \omega_{i j}-\sum_{k} \omega_{i k} \wedge \omega_{k j}=-\frac{1}{2} \sum_{k l} R_{i j k l} \theta_{k} \wedge \theta_{l}
\end{array}\right.
$$

where $\omega_{i j}+\omega_{j i}=0, R_{i j k l}+R_{i j l k}=0$, and $R_{i j k l}$ are the components of the Riemannian curvature tensor of $M$ with respect to the induced metric $d X \cdot d X$.

From $\mathrm{d} e_{i}=\mathrm{d}\left(E_{i} \circ \nu\right)=\nu^{*} \mathrm{~d} E_{i}=\sum_{j} \nu^{*} \theta_{i j} e_{j}-\nu^{*} \theta_{i} \nu$, we get

$$
\left\{\begin{array}{l}
\omega_{i j}=\nu^{*} \theta_{i j} \\
\nu^{*} \theta_{i}=-\sum_{j} h_{i j} \omega_{j},
\end{array}\right.
$$

where $\omega_{i j}+\omega_{j i}=0, h_{i j}=h_{j i}$. 
Let $F: S^{n} \rightarrow \mathbb{R}^{+}$be a smooth function, we denote the coefficients of covariant differential of $F, \operatorname{grad}_{S^{n}} F, D^{2} F$ with respect to $\left\{E_{i}\right\}_{i=1, \cdots, n}$ by $F_{i}, F_{i j}, F_{i j k}$ respectively.

From Ricci identity and (4), we have

$$
F_{i j k}-F_{i k j}=\sum_{m} \tilde{R}_{m i j k}=\delta_{i k} F_{j}-\delta_{i j} F_{k}
$$

where $F_{i j k}$ denote the coefficients of the covariant differential of $F_{i j}$ on $S^{n}$.

So, if we denote the coefficients of $A_{F}$ by $A_{i j}$, then we have from (7)

$$
A_{i j k}=A_{j i k}=A_{i k j}
$$

where $A_{i j k}$ denotes coefficient of the covariant differential of $A_{F}$ on $S^{n}$.

Let $s_{i j}=\sum_{k}\left(A_{i k} \circ \nu\right) h_{k j}, S_{F}=-A_{F} \circ \mathrm{d} \nu$, then we have $S_{F}\left(e_{j}\right)=\sum_{i} s_{i j} e_{i}$. We call $S_{F}$ to be $F$-Weingarten operator. From the positive definite of $\left(A_{i j}\right)$ and the symmetry of $\left(h_{i j}\right)$, we know the eigenvalues of $\left(s_{i j}\right)$ are all real (in fact, because $A=\left(A_{i j}\right)$ is positive definite, there exists a non-singular matrix $C$ such that $A=C^{t} C$, we have $S=\left(s_{i j}\right)=A B$ has the same eigenvalues with the real symmetric matrix $C B C^{T}$, which follows from $|\lambda I-S|=|\lambda I-A B|=\left|\lambda I-C^{t} C B\right|=\left|\lambda I-C B C^{t}\right|$, where $\left.B=\left(h_{i j}\right)\right)$. we call them anisotropic principal curvatures, and denote them by $\lambda_{1}, \cdots, \lambda_{n}$.

We have $n$ invariants, the elementary symmetric function $\sigma_{r}$ of the anisotropic principal curvatures:

$$
\sigma_{r}=\sum_{i_{1}<\cdots i_{r}} \lambda_{i_{1}} \cdots \lambda_{i_{n}} \quad(1 \leq r \leq n)
$$

For convenience, we set $\sigma_{0}=1$. The $r$-anisotropic mean curvature $M_{r}$ is defined by

$$
M_{r}=\sigma_{r} / C_{n}^{r}, \quad C_{n}^{r}=\frac{n !}{r !(n-r) !} .
$$

Using the characteristic polynomial of $S_{F}, \sigma_{r}$ is defined by

$$
\operatorname{det}\left(t I-S_{F}\right)=\sum_{r=0}^{n}(-1)^{r} \sigma_{r} t^{n-r} .
$$

So, we have

$$
\sigma_{r}=\frac{1}{r !} \sum_{i_{1}, \cdots, i_{r} ; j_{1}, \cdots, j_{r}} \delta_{i_{1} \cdots i_{r}}^{j_{1} \cdots j_{r}} s_{i_{1} j_{1}} \cdots s_{i_{r} j_{r}},
$$

where $\delta_{i_{1} \cdots i_{r}}^{j_{1} \cdots j_{r}}$ is the usual generalized Kronecker symbol, i.e., $\delta_{i_{1} \cdots i_{r}}^{j_{1} \cdots j_{r}}$ equals +1 (resp. -1) if $i_{1} \cdots i_{r}$ are distinct and $\left(j_{1} \cdots j_{r}\right)$ is an even (resp. odd) permutation of $\left(i_{1} \cdots i_{r}\right)$ and in other cases it equals zero.

We define $(F \circ \nu)_{i},\left(F_{i} \circ \nu\right)_{j},\left(A_{i j} \circ \nu\right)_{k}$ by

$$
\mathrm{d}(F \circ \nu)=\sum_{i}(F \circ \nu)_{i} \omega_{i},
$$




$$
\begin{gathered}
\mathrm{d}\left(F_{i} \circ \nu\right)+\sum_{j}\left(F_{j} \circ \nu\right) \omega_{j i}=\sum_{j}\left(F_{i} \circ \nu\right)_{j} \omega_{j}, \\
\mathrm{~d}\left(A_{i j} \circ \nu\right)+\sum\left(A_{k j} \circ \nu\right) \omega_{k i}+\sum_{k}\left(A_{i k} \circ \nu\right) \omega_{k j}=\sum_{k}\left(A_{i j} \circ \nu\right)_{k} \omega_{k} .
\end{gathered}
$$

By use of (3), (5) and (6), we have by a direct calculation

$$
\left\{\begin{array}{c}
(F \circ \nu)_{i}=-\sum_{j} h_{i j} F_{j} \circ \nu, \\
\left(F_{i} \circ \nu\right)_{j}=-\sum_{k} h_{j k} F_{i k} \circ \nu, \\
\left(A_{i j} \circ \nu\right)_{k}=-\sum_{l} h_{k l} A_{i j l} \circ \nu .
\end{array}\right.
$$

\section{$\S 3$ Some Lemmas}

We introduce an important operator $P_{r}$ by

$$
P_{r}=\sigma_{r} I-\sigma_{r-1} S_{F}+\cdots+(-1)^{r} S_{F}^{r}, \quad r=0,1, \cdots, n .
$$

We have the following lemmas:

Lemma 3.1. $\left(S_{F} A_{F}\right)^{t}=S_{F} A_{F},\left(\mathrm{~d} \nu \circ S_{F}\right)^{t}=\mathrm{d} \nu \circ S_{F}, s_{i j k}=s_{i k j}, \sum_{l} h_{i l} s_{l k}=\sum_{l} h_{k l} s_{l i}$, $\sum_{l} h_{k l}\left(P_{r}\right)_{l j}=\sum_{l} h_{j l}\left(P_{r}\right)_{l k}$, where $s_{i j k}$ are the components of the covariant derivarive of $s_{i j}$.

Proof. Since $S_{F}=-A_{F} \circ \mathrm{d} \nu$, and $A_{F}, \mathrm{~d} \nu$ are symmetric operators, the first two identities are obvious. From the symmetry property (8) of $A_{i j k}, h_{i j}=h_{j i}$ and Codazzi equation $h_{i j k}=h_{i k j}$, we have by use of (16)

$$
\begin{aligned}
& s_{i j k}=\left(\sum_{l} A_{i l} h_{l j}\right)_{k}=\sum_{l}\left(A_{i l} \circ \nu\right)_{k} h_{l j}+\sum_{l} A_{i l} h_{l j k} \\
&=-\sum_{l, m}\left(A_{i l m} \circ \nu\right) h_{l j} h_{k m}+\sum_{l} A_{i l} h_{l j k} \\
&=\sum_{m}\left(A_{i m} \circ \nu\right)_{j} h_{m k}+\sum_{l} A_{i l} h_{l k j}=\left(\sum_{l} A_{i l} h_{l k}\right)_{j}=s_{i k j} . \\
& \sum_{l} h_{i l} s_{l k}=\sum_{l, m} h_{i l} A_{l m} h_{m k}=\sum_{l, m} h_{k m} A_{m l} h_{l i}=\sum_{l} h_{k l} s_{l i} .
\end{aligned}
$$

By use of the above formula and the definition of $P_{r}$, we get the last identity in Lemma 3.1.

Lemma 3.2. The matrix of $P_{r}$ is given by:

$$
\left(P_{r}\right)_{i j}=\frac{1}{r !} \sum_{i_{1}, \cdots, i_{r} ; j_{1}, \cdots, j_{r}} \delta_{i_{1} \cdots i_{r} j}^{j_{1} \cdots j_{r} i} s_{i_{1} j_{1}} \cdots s_{i_{r} j_{r}}
$$


Proof. We prove Lemma 3.2 inductively. For $r=0$, it is easy to check that (19) is true.

We can check directly

$$
\delta_{i_{1} \cdots i_{q}}^{j_{1} \cdots j_{q}}=\left|\begin{array}{ccccc}
\delta_{i_{1}}^{j_{1}} & \delta_{i_{1}}^{j_{2}} & \cdots & \delta_{i_{1}}^{j_{q-1}} & \delta_{i_{1}}^{j_{q}} \\
\delta_{i_{2}}^{j_{1}} & \delta_{i_{2}}^{j_{2}} & \cdots & \delta_{i_{2}}^{j_{q-1}} & \delta_{i_{2}}^{j_{q}} \\
\vdots & \vdots & \ddots & \vdots & \vdots \\
\delta_{i_{q-1}}^{j_{1}} & \delta_{i_{q-1}}^{j_{2}} & \cdots & \delta_{i_{q_{-1}-1}}^{j_{q}} & \delta_{i_{q-1}}^{j_{q}} \\
\delta_{i_{q}}^{j_{1}} & \delta_{i_{q}}^{j_{2}} & \cdots & \delta_{i_{q}}^{j_{q}} & \delta_{i_{q}}^{j_{q}}
\end{array}\right|
$$

Assume that (19) is true for $r=k$, we only need to show that it is also true for $r=k+1$. For $r=k+1$, Using (12) and (20), we have

$$
\begin{aligned}
& R H S \text { of }(19)=\frac{1}{(k+1) !} \sum_{i_{1}, \cdots, i_{k+1} ; j_{1}, \cdots, j_{k+1}} \delta_{i_{1} \cdots i_{k+1} j}^{j_{1} \cdots j_{k+1} i} s_{i_{1} j_{1}} \cdots s_{i_{k+1} j_{k+1}} \\
& =\frac{1}{(k+1) !} \sum\left|\begin{array}{ccccc}
\delta_{i_{1}}^{j_{1}} & \delta_{i_{1}}^{j_{2}} & \cdots & \delta_{i_{1}}^{j_{k+1}} & \delta_{i_{1}}^{i} \\
\delta_{i_{2}}^{j_{1}} & \delta_{i_{2}}^{j_{2}} & \cdots & \delta_{i_{2}}^{j_{k+1}} & \delta_{i_{2}}^{i} \\
\vdots & \vdots & \ddots & \vdots & \vdots \\
\delta_{i_{k+1}}^{j_{1}} & \delta_{i_{k+1}}^{j_{2}} & \cdots & \delta_{i_{k+1}}^{j_{k+1}} & \delta_{i_{k+1}}^{i} \\
\delta_{j}^{j_{1}} & \delta_{j}^{j_{2}} & \cdots & \delta_{j}^{j_{k+1}} & \delta_{j}^{i}
\end{array}\right| s_{i_{1} j_{1}} \cdots s_{i_{k+1} j_{k+1}} \\
& =\frac{1}{(k+1) !} \sum\left(\delta_{j}^{i} \delta_{i_{1} \cdots i_{k+1}}^{j_{1} \cdots j_{k+1}}-\delta_{j}^{j_{k+1}} \delta_{i_{1} \cdots i_{k} i_{k+1}}^{j_{1} \cdots j_{k} i}+\cdots\right) s_{i_{1} j_{1}} \cdots s_{i_{k+1} j_{k+1}} \\
& =\sigma_{k+1} \delta_{i j}-\frac{1}{(k+1) !} \sum \delta_{j}^{j_{k+1}} \delta_{i_{1} \cdots i_{k} i_{k+1}}^{j_{1} \cdots j_{k} i} s_{i_{1} j_{1}} \cdots s_{i_{k+1} j_{k+1}}+\cdots \\
& =\sigma_{k+1} \delta_{i j}-\sum\left(P_{k}\right)_{i i_{k+1}} s_{i_{k+1} j} \\
& =\left(P_{k+1}\right)_{i j} \text {. }
\end{aligned}
$$

Lemma 3.3. For each $r$, we have

(i). $\sum_{j}\left(P_{r}\right)_{j i j}=0$,

(ii). $\operatorname{tr}\left(P_{r} S_{F}\right)=(r+1) \sigma_{r+1}$,

(iii). $\operatorname{tr}\left(P_{r}\right)=(n-r) \sigma_{r}$.

Proof. (i). Noting $\left(j, j_{r}\right)$ is skew symmetric in $\delta_{i_{1} \cdots i_{r} i}^{j_{1} \cdots j_{r} j}$ and $\left(j, j_{r}\right)$ is symmetric in $s_{i_{1} j_{1}} \cdots s_{i_{r} j_{r} j}$ (from Lemma 3.1), we have

$$
\sum_{j}\left(P_{r}\right)_{j i j}=\frac{1}{(r-1) !} \sum_{i_{1}, \cdots, i_{r} ; j_{1}, \cdots, j_{r} ; j} \delta_{i_{1} \cdots i_{r} i}^{j_{1} \cdots j_{r} j} s_{i_{1} j_{1}} \cdots s_{i_{r} j_{r} j}=0 .
$$

(ii). Using (19) and (12), we have

$$
\begin{aligned}
\operatorname{tr}\left(P_{r} S_{F}\right) & =\sum_{i j}\left(P_{r}\right)_{i j} s_{j i} \\
& =\frac{1}{r !} \sum_{i_{1}, \cdots, i_{r} ; j_{1}, \cdots, j_{r} ; i, j} \delta_{i_{1} \cdots i_{r} j}^{j_{1} \cdots j_{r} i} s_{i_{1} j_{1}} \cdots s_{i_{r} j_{r}} s_{j i} \\
& =(r+1) \sigma_{r+1} .
\end{aligned}
$$


(iii). Using (ii) and the definition of $P_{r}$, we have

$$
\operatorname{tr}\left(P_{r}\right)=\operatorname{tr}\left(\sigma_{r} I\right)-\operatorname{tr}\left(P_{r-1} S_{F}\right)=n \sigma_{r}-r \sigma_{r}=(n-r) \sigma_{r}
$$

Remark 3.1 When $F=1$, Lemma 3.3 was a well-known result (for example, see Barbosa-Colares [1]).

Lemma 3.4. If $\lambda_{1}=\lambda_{2}=\cdots=\lambda_{n}=$ const $\neq 0$, then up to translations and homotheties, $X(M)$ is the Wulff shape.

Proof. Choose a local orthogonal frame $e_{1}, e_{2}, \cdots, e_{n}$ such that $A_{F}$ is diagonalized:

$$
A_{F}=\operatorname{diag}\left(\mu_{1}, \cdots, \mu_{n}\right) \text {, }
$$

where $\mu_{i}>0$ for $i=1, \cdots, n$ by the convexity condition. Then we have $S_{i j}=\mu_{i} h_{i j}$. From (10) and (12), we get

$$
\begin{aligned}
& 0=M_{1}^{2}-M_{2}=\left(\frac{1}{n} \sum_{i} \mu_{i} h_{i i}\right)^{2}-\frac{2}{n(n-1)} \sum_{i<j} \mu_{i} \mu_{j}\left(h_{i i} h_{j j}-h_{i j}^{2}\right) \\
= & \frac{1}{n^{2}(n-1)}\left\{(n-1)\left(\sum_{i} \mu_{i} h_{i i}\right)^{2}-2 n \sum_{i<j} \mu_{i} \mu_{j}\left(h_{i i} h_{j j}-h_{i j}^{2}\right)\right\} \\
= & \frac{1}{n^{2}(n-1)} \sum_{i<j}\left\{\left(\mu_{i} h_{i i}-\mu_{j} h_{j j}\right)^{2}+2 n \mu_{i} \mu_{j} h_{i j}^{2}\right\},
\end{aligned}
$$

so, $\mu_{1} h_{11}=\mu_{2} h_{22}=\cdots=\mu_{n} h_{n n}$ and $h_{i j}=0$ when $i \neq j$. Then, from [10] or [3], [14], up to translations and homotheties, $X(M)$ is the Wulff shape.

\section{$\S 4$ Proofs of Theorem 1.1-Theorem 1.5}

Proof of Theorem 1.1 By use of (5), we have

$$
\langle X, \nu\rangle_{i}=-\sum_{j} h_{i j}\left\langle X, e_{j}\right\rangle, \quad\left\langle X, e_{j}\right\rangle_{i}=\delta_{i j}+h_{i j}\langle X, \nu\rangle
$$

so, from (16), Lemma 3.1 and (i), (ii), (iii) of Lemma 3.3, we have the following calculation

$$
\begin{aligned}
& \operatorname{div}\left\{P_{r}\left(\langle X, \nu\rangle \operatorname{grad}_{S^{n}} F-F \operatorname{grad}|X|^{2} / 2\right)\right\} \\
= & \sum_{i j}\left\{\left(P_{r}\right)_{i j}\left(\langle X, \nu\rangle F_{j}-F\left\langle X, e_{j}\right\rangle\right)\right\}_{i} \\
= & \sum_{i j}\left(P_{r}\right)_{i j}\left\{-\sum_{k} h_{i k}\left(\left\langle X, e_{k}\right\rangle F_{j}+\langle X, \nu\rangle F_{j k}-F_{k}\left\langle X, e_{j}\right\rangle\right)-F \delta_{i j}-F h_{i j}\langle X, \nu\rangle\right\} \\
= & -\sum_{i j k} h_{k i}\left(P_{r}\right)_{i j}\left\langle X, e_{k}\right\rangle F_{j}+\sum_{i j k} h_{k i}\left(P_{r}\right)_{i j}\left\langle X, e_{j}\right\rangle F_{k} \\
& -\langle X, \nu\rangle \sum_{i j k}\left(P_{r}\right)_{i j}\left(F_{j k}+F \delta_{j k}\right) h_{k i}-F \sum_{i}\left(P_{r}\right)_{i i} \\
= & -\sum_{i j k} h_{k i}\left(P_{r}\right)_{i j}\left\langle X, e_{k}\right\rangle F_{j}+\sum_{i j k} h_{j i}\left(P_{r}\right)_{i k}\left\langle X, e_{k}\right\rangle F_{j} \\
& -\langle X, \nu\rangle \sum_{i j k}\left(P_{r}\right)_{i j} A_{j k} h_{k i}-F \sum_{i}\left(P_{r}\right)_{i i} \\
= & -\langle X, \nu\rangle \sum_{i j}\left(P_{r}\right)_{i j} s_{j i}-F \sum_{i}\left(P_{r}\right)_{i i} \\
= & -\langle X, \nu\rangle \operatorname{tr}\left(P_{r} S_{F}\right)-F \operatorname{tr}\left(P_{r}\right) \\
= & -\langle X, \nu\rangle(r+1) \sigma_{r+1}-F(n-r) \sigma_{r} \\
= & -(n-r) C_{n}^{r}\left(F M_{r}+M_{r+1}\langle X, \nu\rangle\right) .
\end{aligned}
$$


Integrating the above formula over $M$, we get (2) by use of Stokes Theorem.

Proof of Theorem 1.2: From (2), we have

$$
\begin{gathered}
\int_{M}\left(F+M_{1}\langle X, \nu\rangle\right) \mathrm{d} A_{X}=0, \\
\int_{M}\left(F M_{1}+M_{2}\langle X, \nu\rangle\right) \mathrm{d} A_{X}=0 .
\end{gathered}
$$

By the assumption $M_{1}=$ constant, we get from (23) and (24)

$$
\int_{M}\langle X, \nu\rangle\left(M_{1}^{2}-M_{2}\right) \mathrm{d} A_{X}=0
$$

On the other hand,

$$
M_{1}^{2}-M_{2}=\frac{1}{n^{2}(n-1)} \sum_{j<i}\left(\lambda_{i}-\lambda_{j}\right)^{2} \geq 0 .
$$

Thus, if $\langle X, \nu\rangle$ has fixed sign, then $M_{1}^{2}-M_{2}=0$, so

$$
\lambda_{1}=\lambda_{2}=\cdots=\lambda_{n}
$$

Thus, from Lemma 3.4, up to translations and homotheties, $X(M)$ is the Wulff shape.

Proof of Theorem 1.3: We have the fact that if $M$ is compact and $M_{r}>0$ then

$$
M_{r-1} \geq M_{r}^{(r-1) / r}, \quad 2 \leq r \leq n
$$

with equality holds if and only if $\lambda_{1}=\lambda_{2}=\cdots=\lambda_{n}$ on $M$ (cf. [9], [2]). Indeed (27) holds if $M_{r} \equiv$ const, since $M$ is compact, there exists a point $p_{0}$ on $M$ such that all principal curvatures are positive at $p_{0}$, so all anisotropic principal curvatures are positive at $p_{0}$. Applying (27) inductively, one sees that if $M_{r} \equiv$ const, then

$$
M_{r} \leq M_{1}^{r},
$$

here again equality holds if and only if $\lambda_{1}=\lambda_{2}=\cdots=\lambda_{n}$.

Integrating $F M_{r}^{(r-1) / r} \leq F M_{r-1}$ over $M$, using (2) and $M_{r}=$ constant, we get

$$
M_{r}^{(r-1) / r} \int_{M} F \mathrm{~d} A_{X} \leq \int_{M} F M_{r-1} \mathrm{~d} A_{X}=-M_{r} \int_{M}\langle X, \nu\rangle \mathrm{d} A_{X} .
$$

On the other hand, our assumption $M_{1}=$ constant (thus $M_{1}>0$ ) and (23) implies

$$
\int_{M}\langle X, \nu\rangle \mathrm{d} A_{X}=-\frac{1}{M_{1}} \int_{M} F d A_{X}
$$

Putting (30) into (29), we get

$$
M_{1}^{r} \leq M_{r} .
$$

Therefore equality holds in (28) and $\lambda_{1}=\lambda_{2}=\cdots=\lambda_{n}$ on $M$. Thus, from Lemma 3.4 , up to translations and homotheties, $X(M)$ is the Wulff shape. 
Proof of Theorem 1.4: From (2), we have

$$
\begin{aligned}
& \int_{M}\left(F M_{k}+M_{k+1}\langle X, \nu\rangle\right) \mathrm{d} A_{X}=0 . \\
& \int_{M}\left(F M_{r}+M_{r+1}\langle X, \nu\rangle\right) \mathrm{d} A_{X}=0 .
\end{aligned}
$$

From the assumptions $\frac{M_{r}}{M_{k}}=$ constant, $\frac{M_{r}}{M_{k}} \times(32)-(33)$ implies

$$
\int_{M}\langle X, \nu\rangle\left(M_{r+1}-\frac{M_{r}}{M_{k}} M_{k+1}\right) \mathrm{d} A_{X}=0
$$

From the convexity of $M$, all the principal curvatures of $M$ are positive, so all the anisotropic principal curvature are positive, we have $M_{l}>0,0 \leq l \leq n$ on $M$. From

$$
M_{k} \cdot M_{k+2} \leq M_{k+1}^{2}, \quad \cdots, \quad M_{r-1} M_{r+1} \leq M_{r}^{2},
$$

where equality holds in one of (35) if and only if $\lambda_{1}=\lambda_{2}=\cdots=\lambda_{n}$, we can check

$$
M_{k} M_{r+1} \leq M_{k+1} M_{r}
$$

that is

$$
M_{r+1}-\frac{M_{r}}{M_{k}} M_{k+1} \leq 0 .
$$

On the other hand, we can choose the position of origin $O$ such that $\langle X, \nu\rangle$ has fixed sign. Thus, from (34) and (36), $M_{k} M_{r+1}=M_{k+1} M_{r}$, so $\lambda_{1}=\lambda_{2}=\cdots=\lambda_{n}$. Thus, from Lemma 3.4, up to translations and homotheties, $X(M)$ is the Wulff shape.

Proof of Theorem 1.5: From proof of Theorem 1.3, we know that there exists a point $p_{0} \in M$ such that the anisotropic principal curvature $\lambda_{i}\left(p_{0}\right)>0,1 \leq i \leq n$. From

$\frac{M_{k}}{M_{n}}=$ constant, we have $\frac{M_{k}}{M_{n}}=\frac{M_{k}}{M_{n}}\left(p_{0}\right)>0$. Thus $M_{n} \neq 0$ on $M$, by the continuity of $\lambda_{i}$, we have $\lambda_{i}>0,1 \leq i \leq n$, on $M$. Therefore all principal curvatures of $M$ are positive on $M$ and $M$ is convex. Theorem 1.5 follows from Theorem 1.4.

\section{References}

[1] J. L. M. Barbosa and A. G. Colares, Stability of hypersurfaces with constant r-mean curvature, Ann. Global Anal. Geom., 15(1997), 277-297.

[2] J. Choe, Sufficient conditions for constant mean curvature surfaces to be round, Math. Ann. 323(2002), 143-156.

[3] U. Clarenz, The Wulff-shape minimizes an anisotropic Willmore functional. Interfaces and Free Boundaries 6, 35-359 (2004). 
[4] G. H. Hardy, J. E. Littlewood, and G. Polya, Inequalities, Cambridge Univ. Press, London, 1934.

[5] C. C. Hsiung, Some integral formulas for closed hypersurfaces, Math. Scand. 2 (1954), 286-294.

[6] M. Koiso and B. Palmer, Geometry and stability of surfaces with constant anisotropic mean curvature, Indiana Univ. Math. J., 54 (2005), 1817-1852.

[7] H. Li, Hypersurfaces with constant scalar curvature in space forms, Math. Ann. 305(1996), 665-672.

[8] H. Li, Global rigidity theorems of hypersurfaces, Ark. Math. 35(1997), 327-351.

[9] S. Montiel and A. Ros, Compact hypersurfaces: The Alexandrov theorem for higher order mean curvatures, in Lawson, B. and Tenenblat, K. (eds), Differential Geometry, Pitman Monographs, Vol. 52, Longman, Essex, 1991, pp. 279-296.

[10] B. Palmer, Stability of the Wulff shape. Proc. Amer. Math. Soc. 126 (1998), 36613667.

[11] Udo Simon, Minkowskische integralformeln und ihre Anwendungen in der Differentialgeometrie in Grossen, Math. Ann. 173(1967), 307-321.

[12] W. Süss, Zur relativen Differentialgeometrie, V., Tôhoku Math. J. 30(1929), 202209.

[13] J. Taylor, Crystalline variational problems, Bull. Amer. Math. Soc., 84(1978), 568-588.

[14] Sven Winklmann. A note on the stability of the Wulff shape. Arch. Math. 87 (2006) 272-279.

[15] K. Yano, Integral formulas in Riemannian geometry, Marcel, Dekker,N. Y, 1970.

Department of Mathematical Sciences

Tsinghua University

Beijing 100084

P. R. China

yjhe@math.tsinghua.edu.cn

hli@math.tsinghua.edu.cn 